\title{
Response of Ferrogels Subjected to an AC Magnetic Field
}

\author{
G. Wang, W. J. Tian, and J. P. Huang* \\ Surface Physics Laboratory (National Key laboratory) and Department of Physics, Fudan University, \\ Shanghai 200433, China
}

Received: January 18, 2006; In Final Form: April 10, 2006

\begin{abstract}
When a ferrogel, which is chemically cross-linked polymer networks swollen with a ferrofluid, consisting of magnetic particles having nonlinear characteristics is subjected to an alternating current (ac) magnetic field, the magnetic response will generally consist of ac fields at frequencies of the higher-order harmonics. By using a perturbation approach, we investigate nonlinear ac responses of ferrogels, under an ac magnetic field either coupled with a dc magnetic field or not. It is shown that it is possible to detect the volume fraction and shape of particles in ferrogels by measuring such ac responses. Our results are very well understood in spectral representation and are favorably compared with the experimental observations of suspensions being beyond ferrogels.
\end{abstract}

\section{Introduction}

In recent decades, polymer gels have become a kind of prospecting material for application in many fields, from soft actuators or micromanipulators in technical fields to guarantee controlled drug release ${ }^{1}$, and artificial muscles ${ }^{2}$ to cancer therapy ${ }^{3}$ or an apparatus for immunoblotting. ${ }^{4}$ The preparation of magnetic-field-sensitive polymer gels, called ferrogels, was reported in the past decade, e.g., see ref 5. A ferrogel is a chemically cross-linked polymer network swollen by a ferrofluid. Ferrofluids (also called magnetic fluids) are a colloidal suspension of monodomain ferromagnetic particles with a typical diameter of $\sim 10 \mathrm{~nm} .{ }^{6-8}$ In ferrogels, the finely distributed ferromagnetic particles are attached to the flexible network chains by adhesive forces. The works on the understanding of magnetic elastic behaviors ${ }^{6,9,10}$ and electric properties ${ }^{11}$ of ferrogels have been done.

In the absence of an external magnetic field, the orientations of the permanent magnetic moments in the particles in ferrogels are rotating ceaselessly, so there is no apparent bulk magnetization. When applying a magnetic field to a ferrogel, the moments tend to align with the field to show magnetization. For an ordinary field strength, the tendency of the moments' alignment can be overcome by thermal agitation. However, as field strength increases, the alignment along the direction of the field is intensified. Eventually, the magnetization achieves a saturation. Let us suppose that an individual monodomain particle in ferrogels carries magnetic moment $m_{0}$. According to the Langevin theory, the average moment of the system under a magnetic field can be written as

$$
\langle m\rangle=m_{0}\left(\operatorname{coth} \alpha-\frac{1}{\alpha}\right)
$$

where the Langevin parameter $\alpha$ is

$$
\alpha=\frac{\mu_{0} m_{0} H}{k_{\mathrm{B}} T}
$$

* Electronic address: jphuang@fudan.edu.cn.
Here, $\mu_{0}$ represents the magnetic permeability of vacuum, $H$ is the strength of an external magnetic field, $k_{\mathrm{B}}$ means the Bolzmann constant, and $T$ denotes the absolute temperature.

Anisotropy is a common phenomenon in most materials, and it can be an intrinsic material property (e.g., induced by the application of fields) or a geometrical anisotropy (e.g., in real applications, the shape of particles may deviate from a perfect spherical shape during fabrication). In this work, we consider the cases in which the particles possess an isotropic material property and they may have an anisotropic geometrical shape, in an attempt to take into account the effect of shape anisotropy.

In the literature, one often considers the magnetic induction $\mathbf{B}$ to be proportional with the field strength $\mathbf{H}$, in accordance with the linear relation between them

$$
\mathbf{B}=\mu \mathbf{H}
$$

where $\mu$ is the (linear) permeability. However, this simple relation is only valid at moderate field intensities. In fact, nonlinear phenomena are a common phenomenon in nature. At higher field intensities, deviations of eq 1 become noticeable. In this case, we need the expression containing an additional nonlinear part

$$
\mathbf{B}=\mu \mathbf{H}+\xi|H|^{2} \mathbf{H} \equiv \tilde{\mu} \mathbf{H}
$$

where $\xi$ is the nonlinear coefficient. For real parameters under our consideration, we may throw off the symbol of absolute value in eq 2 and then obtain the expression for the nonlinear permeability $\tilde{\mu}$

$$
\tilde{\mu}=\mu+\xi H^{2}
$$

In this equation, there is $\xi H^{2} \ll \mu$ (weak nonlinearity). It must be noted that the expression for $\mathbf{B}$ (eq 2) contains only odd powers of $\mathbf{H}$, because a reversal of the direction of $\mathbf{H}$ leads to a reversal of the direction of $\mathbf{B}$, namely

$$
\mathbf{B}(\mathbf{H})=-\mathbf{B}(-\mathbf{H})
$$

When a composite consisting of dielectric (or magnetic) particles having nonlinear characteristics is subjected to an 
alternating current (ac) electric (or magnetic) field, the electric (or magnetic) response will generally consist of ac fields at frequencies of the higher-order harmonics. ${ }^{12-16}$ The main aim of the present paper is to study the effects of the volume fraction and geometric shape of filler particles on the nonlinear ac responses (harmonics) of ferrogels. For this purpose, we will adopt a perturbation approach, which is suitable for weak nonlinearity. ${ }^{15,17,18}$ In detail, the approach will be used to extract the harmonics of the magnetic induction. In this approach, it was well-established that the third-order nonlinear susceptibility can be calculated from the linear field. ${ }^{19}$ Owing to this reason, the contribution from the higher-order nonlinear characteristics will be omitted throughout the paper, see eqs 2 and 36 .

The paper is organized as follows. In section II, we present the formalism for the nonlinear ac responses (harmonics) of ferrogels contaning particles with different shape. In section III, we perform numerical calculations for such responses of the ferrogels under various conditions, and further present an analysis based on spectral representation and a comparison with some existing works. This is followed by a discussion and conclusion in section IV.

\section{Formalism}

A. Nonlinear Characteristics. Let us start by considering a particle with nonlinear permeability $\tilde{\mu}_{1}$ in a ferrogel. By solving the Laplacian equation, the local magnetic field $\mathbf{H}_{1}$ inside the particle is given by

$$
\mathbf{H}_{1}=\frac{3 \mu_{2}}{\tilde{\mu}_{1}+2 \mu_{2}} \mathbf{H}_{0}
$$

where $H_{0}$ is the external applied magnetic field and $\mu_{2}$ represents the permeability of the host. It is worth noting that the host means a cross-linked polymer network plus a liquid. Since both of them can be nonmagnetic in real situations, we set their permeabilities to be equal to each other. (For the numerical calculation in section III, we shall set $\mu_{2}=1$.) Similar to eq 3 , the $\tilde{\mu}_{1}$ in eq 5 denotes the nonlinear permeability of the particle

$$
\tilde{\mu}_{1}=\mu_{1}+\xi H_{1}^{2}
$$

with $\xi$ being the nonlinear coefficient.

B. Nonlinear AC Responses in the Cases Of Spheres. 1 . Harmonics up to the Third Order. In the cases of particles with spherical shape, the effective permeability $\mu_{\mathrm{e}}$ of the whole ferrogel is determined by the Maxwell-Garnett theory ${ }^{20}$

$$
\frac{\mu_{\mathrm{e}}-\mu_{2}}{\mu_{\mathrm{e}}+2 \mu_{2}}=p \frac{\tilde{\mu}_{1}-\mu_{2}}{\tilde{\mu}_{1}+2 \mu_{2}}
$$

where $p$ denotes the volume fraction of particles in the system. Now, the magnetic induction $B$ of the system can be defined as

$$
B=\mu_{\mathrm{e}} H_{0}
$$

In view of the weak nonlinarity (eq 6), for eq 8 we perform a Taylor expansion by taking $\xi H_{0}^{2}$ as a small perturbation and then obtain

$$
\begin{gathered}
B=\frac{H_{0} \mu_{2}\left[\mu_{1}+2 p \mu_{1}-2(p-1) \mu_{2}\right]}{\mu_{1}-p \mu_{1}+(2+p) \mu_{2}}+ \\
\frac{81 H_{0}^{3} p \mu_{2}{ }^{4} \xi}{\left(\mu_{1}+2 \mu_{2}\right)^{2}\left[\mu_{1}-p \mu_{1}+(2+p) \mu_{2}\right]^{2}}+O[\xi]^{2}
\end{gathered}
$$

In case of an external sinusoidal magnetic field of the form

$$
\mathbf{H}_{0}=\mathbf{H}_{\mathrm{ac}}(t) \equiv \mathbf{H}_{\mathrm{ac}} \sin (\omega t)
$$

where $\omega$ means the angular frequency of the field and $t$ the time, the substitution of eq 10 into eq 9 yields

$$
\begin{array}{r}
B=\frac{H_{\mathrm{ac}} \mu_{2}\left[\mu_{1}+2 p \mu_{1}-2(p-1) \mu_{2}\right] \sin (\omega t)}{\mu_{1}-p \mu_{1}+(2+p) \mu_{2}}+ \\
\frac{81 H_{\mathrm{ac}}{ }^{3} p \mu_{2}{ }^{4} \xi \sin (\omega t)^{3}}{\left(\mu_{1}+2 \mu_{2}\right)^{2}\left[\mu_{1}-p \mu_{1}+(2+p) \mu_{2}\right]^{2}}
\end{array}
$$

In this equation, we have neglected $O[\xi]^{2}$. In view of the identity, $\sin ^{3}(\omega t)=(3 / 4) \sin (\omega t)-(1 / 4) \sin (3 \omega t)$, eq 11 can be rewritten as the superposition of odd-order harmonics

$$
B=B_{1} \sin (\omega t)+B_{3} \sin (3 \omega t)
$$

where the first and third harmonics can be given by

$$
\begin{gathered}
B_{1}=\frac{H_{\mathrm{ac}} \mu_{2}\left[\mu_{1}+2 p \mu_{1}-2(p-1) \mu_{2}\right]}{\mu_{1}-p \mu_{1}+(p+2) \mu_{2}}+ \\
\frac{243 H_{\mathrm{ac}}{ }^{3} p \mu_{2}{ }^{4} \xi}{4\left(\mu_{1}+2 \mu_{2}\right)^{2}\left[\mu_{1}-p \mu_{1}+(p+2) \mu_{2}\right]^{2}} \\
B_{3}=-\frac{81 H_{\mathrm{ac}}{ }^{3} p \mu_{2}{ }^{4} \xi}{4\left(\mu_{1}+2 \mu_{2}\right)^{2}\left[\mu_{1}-p \mu_{1}+(p+2) \mu_{2}\right]^{2}}
\end{gathered}
$$

Here, only harmonics up to the third order are extracted analytically.

On the other hand, under the application of a dc field, $\mathbf{H}_{\mathrm{dc}}$, and an ac field, $\mathbf{H}_{\mathrm{ac}}(t)$, namely

$$
\mathbf{H}_{0}(t)=\mathbf{H}_{\mathrm{dc}}+\mathbf{H}_{\mathrm{ac}} \sin (\omega t)
$$

the magnetic induction is

$$
B=\mu_{\mathrm{e}}\left[H_{\mathrm{dc}}+H_{\mathrm{ac}} \sin (\omega t)\right]
$$

In this case, eq 11 is re-expressed as the superposition of the dc component $B_{0}$ and harmonics

$$
B=B_{0}+B_{1} \sin (\omega t)+B_{2} \cos (2 \omega t)+B_{3} \sin (3 \omega t)
$$

where the first, second, and third harmonics, $B_{1}, B_{2}$, and $B_{3}$, are, respectively

$$
\begin{gathered}
B_{1}=\left[\frac{H_{\mathrm{ac}} \mu_{2}\left[\mu_{1}+2 p \mu_{1}-2(p-1) \mu_{2}\right]}{\mu_{1}-p \mu_{1}+(p+2) \mu_{2}}+\right. \\
\frac{243 H_{\mathrm{ac}}{ }^{3} p \mu_{2}{ }^{4} \xi}{4\left(\mu_{1}+2 \mu_{2}\right)^{2}\left[\mu_{1}-p \mu_{1}+(2+p) \mu_{2}\right]^{2}}+ \\
\left.\frac{243 H_{\mathrm{ac}}{ }^{{ }^{\mathrm{dc}}}{ }^{2} p \mu_{2}{ }^{4} \xi}{\left(\mu_{1}+2 \mu_{2}\right)^{2}\left[\mu_{1}-p \mu_{1}+(2+p) \mu_{2}\right]^{2}}\right] \\
B_{2}=-\frac{243 H_{\mathrm{ac}}{ }^{2} H_{\mathrm{dc}} p \mu_{2}{ }^{4} \xi}{2\left(\mu_{1}+\mu_{2}\right)^{2}\left[\mu_{1}-p \mu_{1}+(p+2) \mu_{2}\right]^{2}}
\end{gathered}
$$




$$
B_{3}=-\frac{81 H_{\mathrm{ac}}{ }^{3} p \mu_{2}{ }^{4} \xi}{4\left(\mu_{1}+2 \mu_{2}\right)^{2}\left[\mu_{1}-p \mu_{1}+(2+p) \mu_{2}\right]^{2}}
$$

The expression for $B_{0}$ in eq 17 has been omitted.

2. Higher Harmonics. By using the similar method, higher harmonics can be achieved. Let us take the fifth harmonics as an example. In so doing, we should expand $B$ (eq 8) up to $\xi^{2}$, namely

$$
\begin{array}{r}
B=\frac{H_{0} \mu_{2}\left[\mu_{1}+2 p \mu_{1}-2(p-1) \mu_{2}\right]}{\mu_{1}-p \mu_{1}+(p+2) \mu_{2}}+ \\
\frac{81 H_{0}^{3} p \mu_{2}{ }^{4} \xi}{\left(\mu_{1}+2 \mu_{2}\right)^{2}\left[\mu_{1}-p \mu_{1}+(2+p) \mu_{2}\right]^{2}}+ \\
\frac{729 H_{0}{ }^{5}(p-1) p \mu_{2}{ }^{6} \xi^{2}}{\left(\mu_{1}+2 \mu_{2}\right)^{4}\left[\mu_{1}-p \mu_{1}+(p+2) \mu_{2}\right]^{3}}
\end{array}
$$

When applying a single ac magnetic field (eq 10), we obtain

$$
B=B_{1} \sin (\omega t)+B_{3} \sin (3 \omega t)+B_{5} \sin (5 \omega t)
$$

where the first, third, and fifth harmonics are, respectively

$$
\begin{gathered}
B_{1}=\frac{H_{\mathrm{ac}} \mu_{2}\left[\mu_{1}+2 p \mu_{1}-2(p-1) \mu_{2}\right]}{\mu_{1}-p \mu_{1}+(p+2) \mu_{2}}+ \\
\frac{243 H_{\mathrm{ac}}{ }^{3} p \mu_{2}{ }^{4} \xi}{4\left(\mu_{1}+2 \mu_{2}\right)^{2}\left[\mu_{1}-p \mu_{1}+(p+2) \mu_{2}\right]^{2}}+ \\
\frac{3645 H_{\mathrm{ac}}{ }^{5}(p-1) p \mu_{2}{ }^{6} \xi^{2}}{8\left(\mu_{1}+2 \mu_{2}\right)^{4}\left[\mu_{1}-p \mu_{1}+(p+2) \mu_{2}\right]^{3}} \\
B_{3}=-\frac{81 H_{\mathrm{ac}}{ }^{3} p \mu_{2}{ }^{4} \xi}{4\left(\mu_{1}+2 \mu_{2}\right)^{2}\left[\mu_{1}-p \mu_{1}+(p+2) \mu_{2}\right]^{2}}- \\
\frac{3645 H_{\mathrm{ac}}{ }^{5}(p-1) p \mu_{2}{ }^{6} \xi^{2}}{16\left(\mu_{1}+2 \mu_{2}\right)^{4}\left[\mu_{1}-p \mu_{1}+(p+2) \mu_{2}\right]^{3}} \\
B_{5}=\frac{729 H_{\mathrm{ac}}{ }^{5}(p-1) p \mu_{2}{ }^{6} \xi^{2}}{16\left(\mu_{1}+2 \mu_{2}\right)^{4}\left[\mu_{1}-p \mu_{1}+(p+2) \mu_{2}\right]^{3}}
\end{gathered}
$$

Here, we have used the identity $\sin ^{5}(\omega t)=(5 / 8) \sin (\omega t)-$ $(5 / 16) \sin (3 \omega t)+(1 / 16) \sin (5 \omega t)$.

In the case of a dc field coupled with an ac field (eq 15), the magnetic induction can be expressed as the superposition of the dc component $\langle s c p\rangle B\langle$ mark $\rangle\langle\text { rmark }\rangle_{0}\langle m x\rangle$ and harmonics

$$
\begin{array}{r}
B=B_{0}^{\prime}+B_{1} \sin (\omega t)+B_{2} \cos (2 \omega t)+B_{3} \sin (3 \omega t)+ \\
B_{4} \cos (4 \omega t)+B_{5} \sin (5 \omega t)
\end{array}
$$

where the first, second, third, fourth, and fifth harmonics are, respectively, expressed as

$$
\begin{aligned}
B_{4} & =\frac{3645 H_{\mathrm{ac}}{ }^{4} H_{\mathrm{dc}}(p-1) p \mu_{2}{ }^{6} \xi^{2}}{8\left(\mu_{1}+2 \mu_{2}\right)^{4}\left[\mu_{1}-p \mu_{1}+(p+2) \mu_{2}\right]^{3}} \\
B_{5} & =\frac{729 H_{\mathrm{ac}}{ }^{5}(p-1) p \mu_{2}{ }^{6} \xi^{2}}{16\left(\mu_{1}+2 \mu_{2}\right)^{4}\left[\mu_{1}-p \mu_{1}+(p+2) \mu_{2}\right]^{3}}
\end{aligned}
$$

$$
\begin{aligned}
& B_{1}=\frac{H_{\mathrm{ac}} \mu_{2}\left[\mu_{1}+2 p \mu_{1}-2(p-1) \mu_{2}\right]}{\mu_{1}-p \mu_{1}+(p+2) \mu_{2}}+ \\
& \quad \frac{243 H_{\mathrm{ac}} H_{\mathrm{dc}}{ }^{2} p \mu_{2}{ }^{4} \xi}{\left(\mu_{1}+2 \mu_{2}\right)^{2}\left[\mu_{1}-p \mu_{1}+(p+2) \mu_{2}\right]^{2}}+ \\
& \frac{3645 H_{\mathrm{ac}}{ }^{5}(p-1) p \mu_{2}{ }^{6} \xi^{2}}{8\left(\mu_{1}+2 \mu_{2}\right)^{4}\left[\mu_{1}-p \mu_{1}+(p+2) \mu_{2}\right]^{3}}+ \\
& \frac{3645 H_{\mathrm{ac}} H_{\mathrm{dc}}{ }^{4}(p-1) p \mu_{2}{ }^{6} \xi^{2}}{\left(\mu_{1}+2 \mu_{2}\right)^{4}\left[\mu_{1}-p \mu_{1}+(p+2) \mu_{2}\right]^{3}}+\frac{3}{4} \\
& {\left[\frac{81 H_{\mathrm{ac}}{ }^{3} p \mu_{2}{ }^{4} \xi}{\left(\mu_{1}+2 \mu_{2}\right)^{2}\left[\mu_{1}-p \mu_{1}+(p+2) \mu_{2}\right]^{2}}+\right.} \\
&\left.\frac{7290 H_{\mathrm{ac}}{ }^{3} H_{\mathrm{dc}}{ }^{2}(p-1) p \mu_{2}{ }^{6} \xi^{2}}{\left(\mu_{1}+2 \mu_{2}\right)^{4}\left[\mu_{1}-p \mu_{1}+(p+2) \mu_{2}\right]^{3}}\right]
\end{aligned}
$$

$$
\begin{aligned}
& B_{2}=- \frac{3645 H_{\mathrm{ac}}{ }^{4} H_{\mathrm{dc}}(p-1) p \mu_{2}{ }^{6} \xi^{2}}{2\left(\mu_{1}+2 \mu_{2}\right)^{4}\left[\mu_{1}-p \mu_{1}+(p+2) \mu_{2}\right]^{3}}+ \\
& \frac{1}{2}\left[-\frac{243 H_{\mathrm{ac}}{ }^{2} H_{\mathrm{dc}} p \mu_{2}{ }^{4} \xi}{\left(\mu_{1}+2 \mu_{2}\right)^{2}\left[\mu_{1}-p \mu_{1}+(p+2) \mu_{2}\right]^{2}}-\right. \\
&\left.\frac{7290 H_{\mathrm{ac}}{ }^{2} H_{\mathrm{dc}}{ }^{3}(p-1) p \mu_{2}{ }^{6} \xi^{2}}{\left(\mu_{1}+2 \mu_{2}\right)^{4}\left[\mu_{1}-p \mu_{1}+(p+2) \mu_{2}\right]^{3}}\right]
\end{aligned}
$$

$$
\begin{gathered}
B_{3}=-\frac{3645 H_{\mathrm{ac}}{ }^{5}(p-1) p \mu_{2}{ }^{6} \xi^{2}}{16\left(\mu_{1}+2 \mu_{2}\right)^{4}\left[\mu_{1}-p \mu_{1}+(p+2) \mu_{2}\right]^{3}}-\frac{1}{4} \\
{\left[\frac{81 H_{\mathrm{ac}}{ }^{3} p \mu_{2}{ }^{4} \xi}{\left(\mu_{1}+2 \mu_{2}\right)^{2}\left[\mu_{1}-p \mu_{1}+(p+2) \mu_{2}\right]^{2}}+\right.} \\
\left.\frac{7290 H_{\mathrm{ac}}{ }^{3} H_{\mathrm{dc}}{ }^{2}(p-1) p \mu_{2}{ }^{6} \xi^{2}}{\left(\mu_{1}+2 \mu_{2}\right)^{4}\left[\mu_{1}-p \mu_{1}+(p+2) \mu_{2}\right]^{3}}\right]
\end{gathered}
$$

The expression for $B_{0}^{\prime}$ in eq 20 has also been omitted. In the above derivation, we have used the identities $\sin ^{2}(\omega t)=[1-$ $\cos (2 \omega t)] / 2$ and $\sin ^{4}(\omega t)=[3-4 \cos (2 \omega t)+\cos (4 \omega t)] / 8$.

By using the similar method, much higher harmonics, e.g., the sixth (if any), seventh, and so forth, can be extracted as well.

C. Nonlinear AC Responses in the Cases of Prolate and Oblate Spheroids. 1. Harmonics up to the Third Order. In section II.B, the spherical shape of particles has been investigated. Now, we are in a position to take into account the shape effect by investigating nonspheres such as prolate and oblate spheroids. To describe the shape of spheroids, we resort to the demagnetizing factor $g .{ }^{21}$ Let us see $a, b$, and $c$ as three principal axes of the spheroids. For a prolate spheroid, there is $a=b<$ $c$, and we have the longitudinal demagnetizing factor $g_{\mathrm{L}}$ along the major axis $c$ given by ${ }^{21}$

$$
g_{\mathrm{L}}=\frac{1}{k^{2}-1}\left[\frac{k}{\sqrt{k^{2}-1}} \ln \left(k+\sqrt{k^{2}-1}\right)-1\right]
$$

with

$$
k=\frac{c}{a}
$$

In this case, there is always $g_{\mathrm{L}}<1 / 3$. For an oblate spheroid with $a<b=c$, the longitudinal demagnetizing factor $g_{\mathrm{L}}$ along 
the major axis $a$ is ${ }^{21}$

$$
g_{\mathrm{L}}=\frac{1}{2}-\frac{1}{4}\left[\frac{k^{2}}{\sqrt{[3] k^{2}-1}} \arcsin \frac{\sqrt{k^{2}-1}}{k}-\frac{1}{k^{2}-1}\right]
$$

In this case, $g_{\mathrm{L}}$ is always bigger than $1 / 3 .{ }^{22}$ There is a sum rule for the factors $g_{\mathrm{L}}+2 g_{\mathrm{T}}=1,{ }^{21}$ where $g_{\mathrm{T}}$ denotes the transverse demagnetizing factor along the minor axis. ${ }^{18,21,23}$ It is worth mentioning that $g_{\mathrm{L}}=g_{\mathrm{T}}=1 / 3$ denotes the spherical shape of particles, which has actually been discussed in section II.B.

For spheroid particles, the solution of the Laplacian equation yields the local magnetic field strength $\mathbf{H}_{1}$ as

$$
\mathbf{H}_{1}=\frac{\mu_{2}}{\mu_{2}+g_{\mathrm{L}}\left(\tilde{\mu}_{1}-\mu_{2}\right)} \mathbf{H}_{0}
$$

The substitution of $g_{\mathrm{L}}=1 / 3$ (spherical shape) into eq 24 reduces to eq 5 , as expected. Then, the effective permeability $\mu_{\mathrm{e}}$ of the ferrogel may be determined by the Maxwell-Garnett equation $^{20,24}$

$$
\frac{g_{\mathrm{L}}\left(\mu_{\mathrm{e}}-\mu_{2}\right)}{\mu_{2}+g_{\mathrm{L}}\left(\mu_{\mathrm{e}}-\mu_{2}\right)}=p \frac{g_{\mathrm{L}}\left(\tilde{\mu}_{1}-\mu_{2}\right)}{\mu_{2}+g_{\mathrm{L}}\left(\tilde{\mu}_{1}-\mu_{2}\right)}
$$

where $\tilde{\mu}_{1}=\mu_{1}+\xi H_{1}{ }^{2}$. Equation 25 is valid for the case in which the particles under discussion are randomly embedded but their orientations are all along the direction of the external field. For prolate spheroids, this can be easily satisfied because the particles along the major axis $c$ are more magnetizable than along the other axes $a$ or $b$. That is, prolate spheroids can easily be aligned with its major axis $c$ along the direction of the external field. For completeness, we shall also numerically calculate oblate spheroids in section III. By combining eq 24 and eq 25, after doing a Taylor expansion by taking $\xi H_{0}^{2}$ as a small perturbation, the magnetic induction $B=\mu_{\mathrm{e}} H_{0}$ admits

$$
\begin{aligned}
B= & \frac{H_{0} \mu_{2}\left[p \mu_{1}+\mu_{2}-p \mu_{2}+g_{\mathrm{L}}(p-1)\left(\mu_{2}-\mu_{1}\right)\right]}{\mu_{2}+g_{\mathrm{L}}(p-1)\left(\mu_{2}-\mu_{1}\right)}+ \\
& \frac{H_{0}{ }^{3} p \mu_{2}{ }^{4} \xi}{\left[g_{\mathrm{L}}\left(\mu_{1}-\mu_{2}\right)+\mu_{2}\right]^{2}\left[-g_{\mathrm{L}}(p-1)\left(\mu_{1}-\mu_{2}\right)+\mu_{2}\right]^{2}}+O[\xi]^{2}
\end{aligned}
$$

When applying a sinusoidal ac field (eq 10) to the system, according to eq 26 , the magnetic induction $B$ can be rewritten as the superposition of harmonics up to the third order

$$
B=B_{1} \sin (\omega t)+B_{3} \sin (3 \omega t)
$$

where the first and third harmonics are, respectively

$$
\begin{aligned}
B_{1}=\frac{H_{\mathrm{ac}} \mu_{2}\left[p\left(\mu_{1}-\mu_{2}\right)+\mu_{2}+g_{\mathrm{L}}(p-1)\left(\mu_{2}-\mu_{1}\right)\right]}{\mu_{2}+g_{\mathrm{L}}(p-1)\left(\mu_{2}-\mu_{1}\right)}+ \\
\frac{3 H_{\mathrm{ac}}{ }^{3} p \mu_{2}{ }^{4} \xi}{4\left[g_{\mathrm{L}}\left(\mu_{1}-\mu_{2}\right)+\mu_{2}\right]^{2}\left[-g_{\mathrm{L}}(p-1)\left(\mu_{1}-\mu_{2}\right)+\mu_{2}\right]^{2}} \\
B_{3}=-\frac{H_{\mathrm{ac}}{ }^{3} p \mu_{2}{ }^{4} \xi}{4\left[g_{\mathrm{L}}\left(\mu_{1}-\mu_{2}\right)+\mu_{2}\right]^{2}\left[-g_{\mathrm{L}}(p-1)\left(\mu_{1}-\mu_{2}\right)+\mu_{2}\right]^{2}}
\end{aligned}
$$

In the presence of external dc and ac fields (eq 15), the magnetic induction is expressed as the superposition of the dc component $B_{0}{ }^{N}$ and harmonics

$$
B=B_{0}{ }^{N}+B_{1} \sin (\omega t)+B_{2} \cos (2 \omega t)+B_{3} \sin (3 \omega t)
$$

where the first, second, and third harmonics are, respectively, given by

$$
\begin{gathered}
B_{1}=\frac{H_{\mathrm{ac}} \mu_{2}\left[p\left(\mu_{1}-\mu_{2}\right)+\mu_{2}+g_{\mathrm{L}}(p-1)\left(\mu_{2}-\mu_{1}\right)\right]}{\mu_{2}+g_{\mathrm{L}}(p-1)\left(\mu_{2}-\mu_{1}\right)}+ \\
\frac{3 H_{\mathrm{ac}}{ }^{3} p \mu_{2}{ }^{4} \xi}{4\left[g_{\mathrm{L}}\left(\mu_{1}-\mu_{2}\right)+\mu_{2}\right]^{2}\left[-g_{\mathrm{L}}(p-1)\left(\mu_{1}-\mu_{2}\right)+\mu_{2}\right]^{2}}+ \\
\frac{3 H_{\mathrm{ac}} H_{\mathrm{dc}}{ }^{2} p \mu_{2}{ }^{4} \xi}{\left[g_{\mathrm{L}}\left(\mu_{1}-\mu_{2}\right)+\mu_{2}\right]^{2}\left[-g_{\mathrm{L}}(p-1)\left(\mu_{1}-\mu_{2}\right)+\mu_{2}\right]^{2}} \\
B_{2}=-\frac{3 H_{\mathrm{ac}}{ }^{2} H_{\mathrm{dc}} p \mu_{2}{ }^{4} \xi}{2\left[g_{\mathrm{L}}\left(\mu_{1}-\mu_{2}\right)+\mu_{2}\right]^{2}\left[-g_{\mathrm{L}}(p-1)\left(\mu_{1}-\mu_{2}\right)+\mu_{2}\right]^{2}} \\
B_{3}=-\frac{H_{\mathrm{ac}}{ }^{3} p \mu_{2}{ }^{4} \xi}{4\left[g_{\mathrm{L}}\left(\mu_{1}-\mu_{2}\right)+\mu_{2}\right]^{2}\left[-g_{\mathrm{L}}(p-1)\left(\mu_{1}-\mu_{2}\right)+\mu_{2}\right]^{2}}
\end{gathered}
$$

The analytic expression for $B_{0}{ }^{N}$ in eq 29 has been omitted.

2. Higher Harmonics. Also, the analytic expressions for higher harmonics can be achieved by including the shape effect. Again, we take the fifth harmonics as an example. In case of an external ac magnetic field (eq 10), similar to what we have done in section II.B.2, we obtain

$$
B=B_{1} \sin (\omega t)+B_{3} \sin (3 \omega t)+B_{5} \sin (5 \omega t)
$$

where the first, third, and fifth harmonics are, respectively, given by

$$
\begin{gathered}
B_{1}=\frac{H_{\mathrm{ac}} \mu_{2}\left[p\left(\mu_{1}+\mu_{2}\right)-\mu_{2}+g_{\mathrm{L}}(p-1)\left(\mu_{2}-\mu_{1}\right)\right]}{\mu_{2}+g_{1}(p-1)\left(\mu_{2}-\mu_{1}\right)}+ \\
\frac{3 H_{\mathrm{ac}}{ }^{3} p \mu_{2}{ }^{4} \xi}{4\left[g_{\mathrm{L}}\left(\mu_{1}-\mu_{2}\right)+\mu_{2}\right]^{2}\left[-g_{\mathrm{L}}(p-1)\left(\mu_{1}-\mu_{2}\right)+\mu_{2}\right]^{2}}+ \\
\frac{5 g_{\mathrm{L}} H_{\mathrm{ac}}{ }^{5}(p-1) p \mu_{2}{ }^{6} \xi^{2}}{8\left[\left(-g_{\mathrm{L}}(p-1)\left(\mu_{1}-\mu_{2}\right)+\mu_{2}\right]^{3}\left[g_{\mathrm{L}}\left(\mu_{1}-\mu_{2}\right)+\mu_{2}\right]^{4}\right.} \\
B_{3}=-\frac{H_{\mathrm{ac}}{ }^{3} p \mu_{2}{ }^{4} \xi}{4\left[g_{\mathrm{L}}\left(\mu_{1}-\mu_{2}\right)+\mu_{2}\right]^{2}\left[-g_{\mathrm{L}}(p-1)\left(\mu_{1}-\mu_{2}\right)+\mu_{2}\right]^{2}}- \\
\frac{5 g_{\mathrm{L}} H_{\mathrm{ac}}{ }^{5}(p-1) p \mu_{2}{ }^{6} \xi^{2}}{16\left[\left(g_{\mathrm{L}}(p-1)\left(\mu_{1}-\mu_{2}\right)-\mu_{2}\right]^{3}\left[g_{\mathrm{L}}\left(\mu_{1}-\mu_{2}\right)+\mu_{2}\right]^{4}\right.} \\
B_{5}=\frac{g_{\mathrm{L}} H_{\mathrm{ac}}{ }^{5}(p-1) p \mu_{2}{ }^{6} \xi^{2}}{16\left[\left(g_{\mathrm{L}}(p-1)\left(\mu_{1}-\mu_{2}\right)-\mu_{2}\right]^{3}\left[g_{\mathrm{L}}\left(\mu_{1}-\mu_{2}\right)+\mu_{2}\right]^{4}\right.}
\end{gathered}
$$

If we apply a dc and an ac magnetic field (eq 15), we obtain the magnetic induction of the system as the superposition of the dc component $B_{0} \mathrm{~S}$ and harmonics 


$$
\begin{array}{r}
B=B_{0}{ }^{\mathrm{S}}+B_{1} \sin (\omega t)+B_{2} \cos (2 \omega t)+B_{3} \sin (3 \omega t)+ \\
B_{4} \cos (4 \omega t)+B_{5} \sin (5 \omega t)
\end{array}
$$

where the expressions for the first, second, third, fourth, and fifth harmonics are, respectively, given by

$$
\begin{aligned}
& B_{1}= \frac{H_{\mathrm{ac}} \mu_{2}\left[p\left(\mu_{1}-\mu_{2}\right)+\mu_{2}+g_{\mathrm{L}}(p-1)\left(\mu_{2}-\mu_{1}\right)\right]}{\mu_{2}+g_{1}(p-L)\left(\mu_{2}-\mu_{1}\right)}+ \\
& \frac{3 H_{\mathrm{ac}} H_{\mathrm{dc}}{ }^{2} p \mu_{2}{ }^{4} \xi}{\left[g_{\mathrm{L}}\left(\mu_{1}-\mu_{2}\right)+\mu_{2}\right]^{2}\left[-g_{\mathrm{L}}(p-1)\left(\mu_{1}-\mu_{2}\right)+\mu_{2}\right]^{2}}+ \\
& \frac{5 g_{1} H_{\mathrm{ac}}{ }^{5}(p-1) p \mu_{2}{ }^{6} \xi^{2}}{8\left[g_{\mathrm{L}}\left(\mu_{1}-\mu_{2}\right)+\mu_{2}\right]^{4}\left[-g_{\mathrm{L}}(p-1)\left(\mu_{1}-\mu_{2}\right)+\mu_{2}\right]^{3}}+ \\
& \frac{5 g_{\mathrm{L}} H_{\mathrm{ac}} H_{\mathrm{dc}}{ }^{4}(p-1) p \mu_{2}{ }^{6} \xi^{2}}{\left[g_{\mathrm{L}}\left(\mu_{1}-\mu_{2}\right)+\mu_{2}\right]^{4}\left[-g_{\mathrm{L}}(p-1)\left(\mu_{1}-\mu_{2}\right)+\mu_{2}\right]^{3}}+ \\
& \frac{3}{4}\left[\frac{H_{\mathrm{ac}}{ }^{3} p \mu_{2}{ }^{4} \xi}{\left[g_{\mathrm{L}}\left(\mu_{1}-\mu_{2}\right)+\mu_{2}\right]^{2}\left[-g_{\mathrm{L}}(p-1)\left(\mu_{1}-\mu_{2}\right)+\mu_{2}\right]^{2}}+\right. \\
& B_{2}= \\
&\left.\frac{10 g_{1} H_{\mathrm{ac}}{ }^{3} H_{\mathrm{dc}}{ }^{2}(p-1) p \mu_{2}{ }^{6} \xi^{2}}{2\left[\frac{1 g^{2}}{2\left[g_{\mathrm{L}}(p-1)\left(\mu_{1}-\mu_{2}\right)-\mu_{2}\right]^{3}\left[g_{\mathrm{L}}\left(\mu_{1}-\mu_{2}\right)+\mu_{2}\right]^{4}}+\mu_{2}\right]^{4}\left[-g_{\mathrm{L}}(p-1)\left(\mu_{1}-\mu_{2}\right)+\mu_{2}\right]^{3}}\right] \\
&-\frac{3 H_{\mathrm{ac}}{ }^{2} H_{\mathrm{dc}} p \mu_{2}{ }^{4} \xi}{\left[g_{\mathrm{L}}\left(\mu_{1}-\mu_{2}\right)+\mu_{2}\right]^{2}\left[-g_{\mathrm{L}}(p-1)\left(\mu_{1}-\mu_{2}\right)+\mu_{2}\right]^{2}}- \\
&\left.\frac{10 g_{\mathrm{L}} H_{\mathrm{ac}}{ }^{2} H_{\mathrm{dc}}{ }^{3}(p-1) p \mu_{2}{ }^{6} \xi^{2}}{\left[-g_{\mathrm{L}}(p-1)\left(\mu_{1}-\mu_{2}\right)-\mu_{2}\right]^{3}\left[g_{\mathrm{L}}\left(\mu_{1}-\mu_{2}\right)+\mu_{2}\right]^{4}}\right]
\end{aligned}
$$$$
B_{3}=-
$$

$$
\begin{gathered}
\frac{5 g_{\mathrm{L}} H_{\mathrm{ac}}{ }^{5}(p-1) p \mu_{2}{ }^{6} \xi^{2}}{16\left[g_{\mathrm{L}}\left(\mu_{1}-\mu_{2}\right)+\mu_{2}\right]^{4}\left[-g_{\mathrm{L}}(p-1)\left(\mu_{1}-\mu_{2}\right)+\mu_{2}\right]^{3}}-\frac{1}{4} \\
{\left[\frac{H_{\mathrm{ac}}{ }^{3} p \mu_{2}{ }^{4} \xi}{\left[g_{\mathrm{L}}\left(\mu_{1}-\mu_{2}\right)+\mu_{2}\right]^{2}\left[-g_{\mathrm{L}}(p-1)\left(\mu_{1}-\mu_{2}\right)+\mu_{2}\right]^{2}}+\right.} \\
\left.\frac{10 g_{1} H_{\mathrm{ac}}{ }^{3} H_{\mathrm{dc}}{ }^{2}(p-1) p \mu_{2}{ }^{6} \xi^{2}}{\left[g_{\mathrm{L}}\left(\mu_{1}-\mu_{2}\right)+\mu_{2}\right]^{4}\left[-g_{\mathrm{L}}(p-1)\left(\mu_{1}-\mu_{2}\right)+\mu_{2}\right]^{3}}\right] \\
B_{4}=-\frac{5 g_{\mathrm{L}} H_{\mathrm{ac}}{ }^{4} H_{\mathrm{dc}}(p-1) p \mu_{2}{ }^{6} \xi^{2}}{8\left[g_{\mathrm{L}}(p-1)\left(\mu_{1}-\mu_{2}\right)-\mu_{2}\right]^{3}\left[g_{\mathrm{L}}\left(\mu_{1}-\mu_{2}\right)+\mu_{2}\right]^{4}} \\
B_{5}=\frac{g_{\mathrm{L}} H_{\mathrm{ac}}{ }^{5}(p-1) p \mu_{2}{ }^{6} \xi^{2}}{16\left[g_{\mathrm{L}}\left(\mu_{1}-\mu_{2}\right)+\mu_{2}\right]^{4}\left[-g_{\mathrm{L}}(p-1)\left(\mu_{1}-\mu_{2}\right)+\mu_{2}\right]^{3}}
\end{gathered}
$$

The analytic expression for $B_{0} S$ in eq 34 has been omitted again.

\section{Numerical Results and Comparison with Some Existing Works}

For the numerical calculations, without loss of generality, we take the following parameters: $p=0.08, \mu_{1}=6$, and $\mu_{2}=1$. Figures 1 and 2 show that, as nonlinear characteristics increase, the harmonics are caused to increase accordingly. This is in agreement with the results obtained in ref 25 in which a nonlinear electrorheological fluid was investigated by using a model of a pair of touching particles for which the multipolar
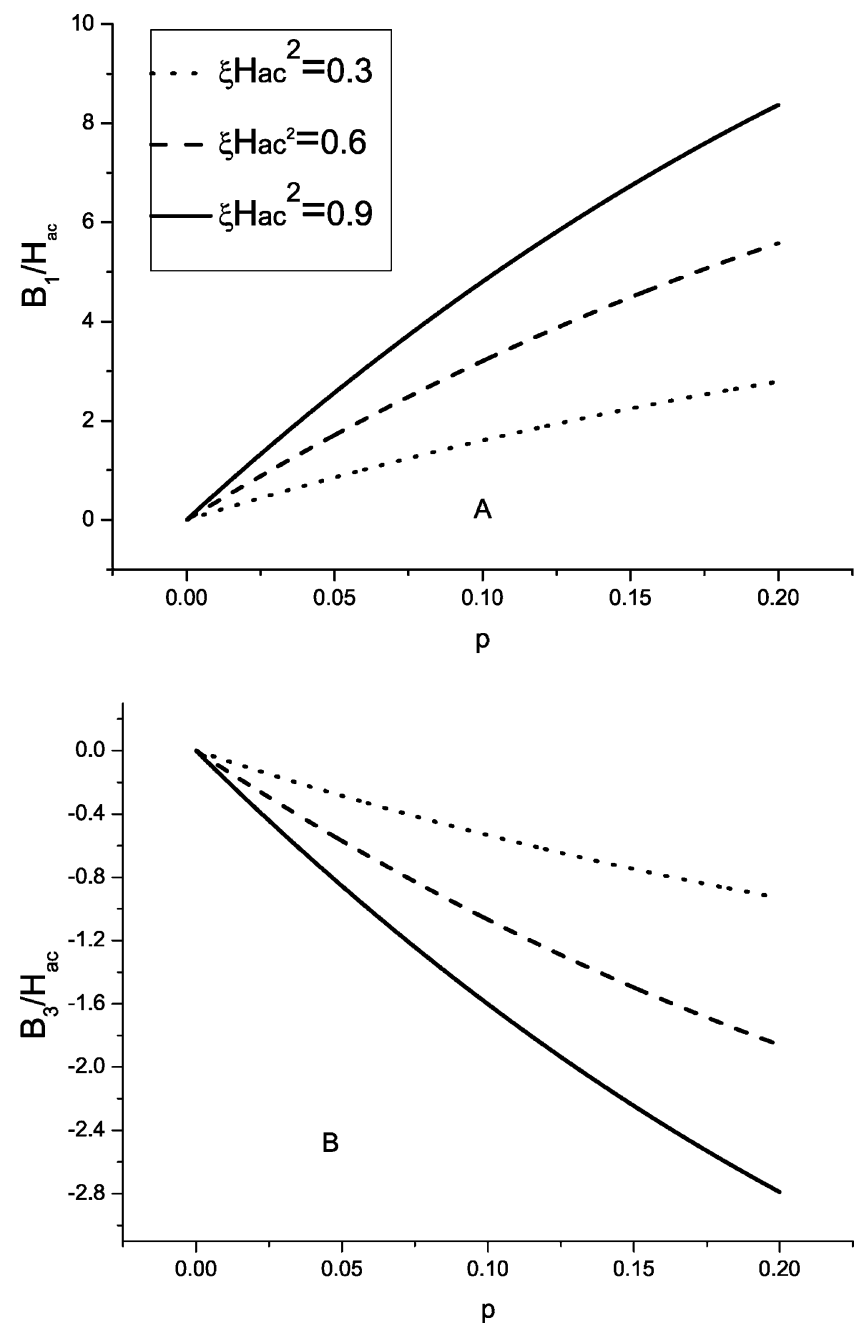

Figure 1. Cases of spherical particles: ac field. Fundamental and third harmonics of magnetic induction $B$ versus volume fraction $p$ for different intrinsic nonlinear characteristic $\xi H_{\mathrm{ac}}^{2}$ according to eq 12 .

interaction was included. We also find that increasing volume fraction of the particles leads to increasing harmonics as well (Figures 1 and 2). The reason is that in the system of interest only particles are assumed to be nonlinear while the host is linear. As the volume fraction increases, the nonlinear component within the system increases naturally, which yields increasing harmonics accordingly.

For the cases of prolate spheroidal particles, increasing aspect ratio $k=c / a$ causes the harmonics to be increased; see Figures 3 and 4. Inverse behavior appears for the cases of oblate spheroidal particles in which increasing aspect ratio $k$ leads to decreasing harmonics; see Figures 5 and 6 . This can be understood as follows. In detail, eq 21 for prolate spheroids shows that increasing $k=c / a$ leads to decreasing $g_{\mathrm{L}}$, whereas eq 23 for oblate spheroids shows that increasing $k=c / a$ leads to increasing $g_{\mathrm{L}}$. We partially explained the behaviors of the harmonic responses by the local field effects in anisotropic dielectric composites, aided by the spectral representation; see ref 23. This is because the spectral representation can reveal the dominant contribution through the self-consistent approach. ${ }^{26}$ This representation can also be developed to deal with the present system. Let us define a material parameter $s=(1-$ $\left.\mu_{1} / \mu_{2}\right)^{-1}$. (Owing to the parameters in use $\mu_{1}=6$ and $\mu_{2}=1$, we obtain $s=-0.2$.) The substitution of this into the expression 

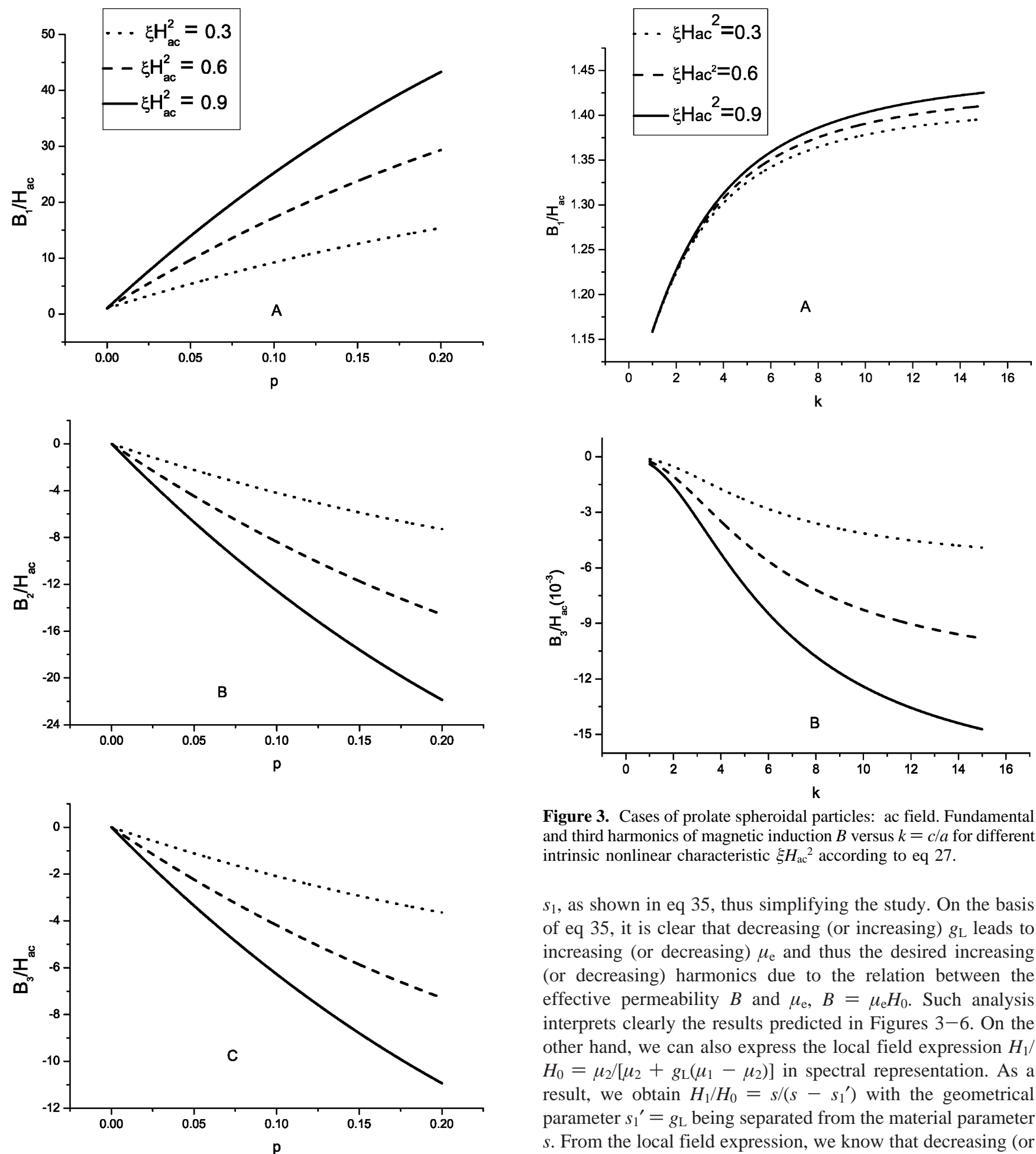

Figure 2. Cases of spherical particles: ac and dc fields. Fundamental, second, and third harmonics of magnetic induction $B$ versus volume fraction $p$ for different intrinsic nonlinear characteristic $\xi H_{\mathrm{ac}}{ }^{2}$ according to eq 17.

for the effective (linear) permeability $\left(\mu_{\mathrm{e}}-\mu_{2}\right) /\left[\mu_{2}+g_{\mathrm{L}}\left(\mu_{\mathrm{e}}-\right.\right.$ $\left.\left.\mu_{2}\right)\right]=p\left(\mu_{1}-\mu_{2}\right) /\left[\mu_{2}+g_{\mathrm{L}}\left(\mu_{1}-\mu_{2}\right)\right]$ yields

$$
\mu_{\mathrm{e}}=\mu_{2}\left[1-\frac{p}{s-s_{1}}\right]
$$

with the geometrical parameter $s_{1}=g_{\mathrm{L}}(1-p)$. From eq 35, it is evident to see that the spectral representation helps to separate the material parameter $s$ from the geometrical parameters, $p$ and

Figure 3. Cases of prolate spheroidal particles: ac field. Fundamental and third harmonics of magnetic induction $B$ versus $k=c / a$ for different intrinsic nonlinear characteristic $\xi H_{\mathrm{ac}}^{2}$ according to eq 27.

$s_{1}$, as shown in eq 35 , thus simplifying the study. On the basis of eq 35 , it is clear that decreasing (or increasing) $g_{\mathrm{L}}$ leads to increasing (or decreasing) $\mu_{\mathrm{e}}$ and thus the desired increasing (or decreasing) harmonics due to the relation between the effective permeability $B$ and $\mu_{\mathrm{e}}, B=\mu_{\mathrm{e}} H_{0}$. Such analysis interprets clearly the results predicted in Figures $3-6$. On the other hand, we can also express the local field expression $H_{1} /$ $H_{0}=\mu_{2} /\left[\mu_{2}+g_{\mathrm{L}}\left(\mu_{1}-\mu_{2}\right)\right]$ in spectral representation. As a result, we obtain $H_{1} / H_{0}=s /\left(s-s_{1}{ }^{\prime}\right)$ with the geometrical parameter $s_{1}{ }^{\prime}=g_{\mathrm{L}}$ being separated from the material parameter $s$. From the local field expression, we know that decreasing (or increasing) $g_{\mathrm{L}}$ leads to increasing (or decreasing) local fields. Such results show that the dependence of harmonics on the shape of particles is originally due to the change in local fields.

The perturbation approach, adopted in this work and presented in ref 17 , has been used by several groups to study nonlinear ac responses of composite materials, e.g., see refs 15 and 16. Regarding the experimental investigation on nonlinear ac responses (harmonics) in suspensions, Klingenberg ${ }^{27}$ has studied such responses of electrorheological suspensions that contain polarizable dielectric particles embedded in a host liquid like silicone oil, and he experimentally showed that the harmonics of the electric current is caused to increase while the external electric field increases. Because of the mathematical similarity 

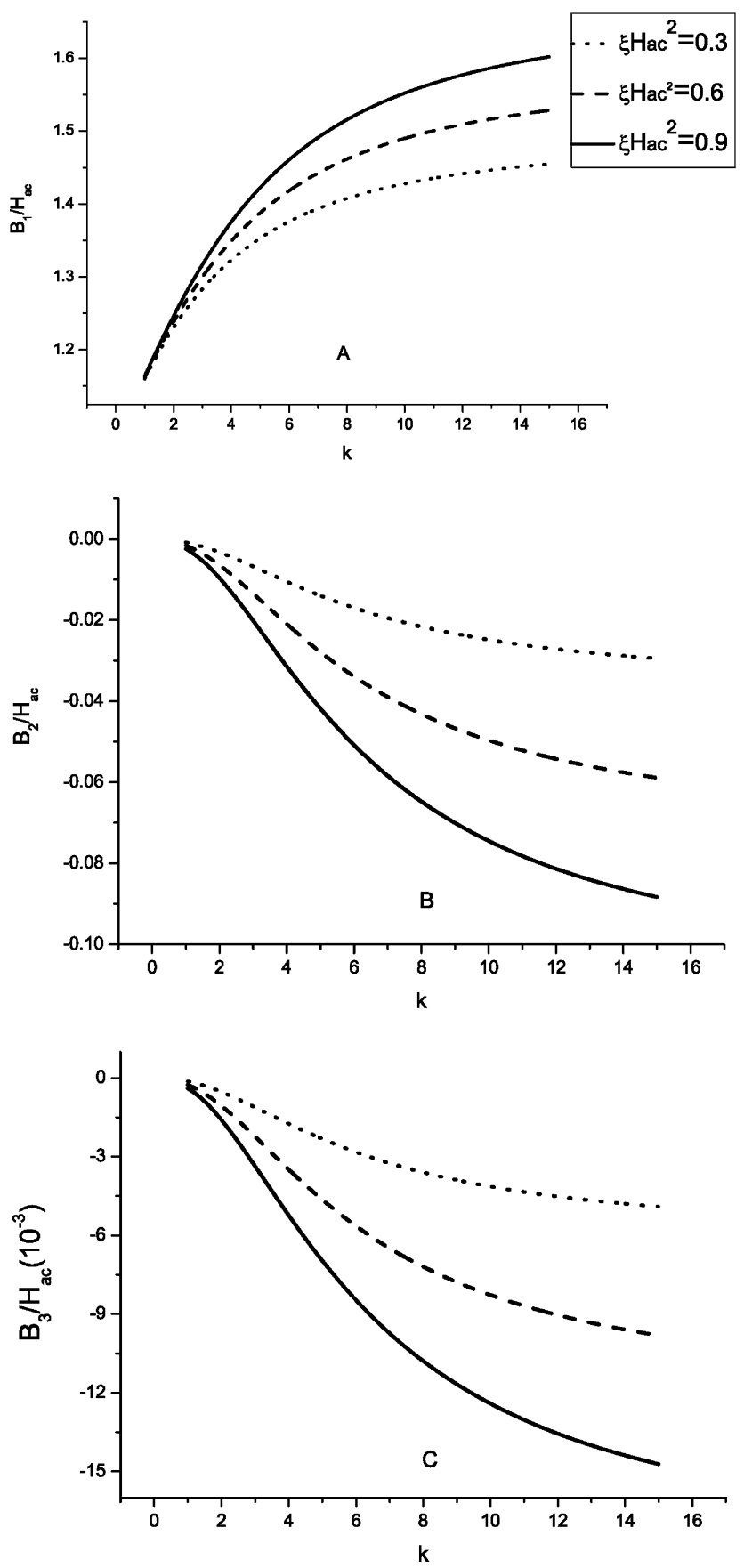

Figure 4. Cases of prolate spheroidal particles: ac and dc fields. Fundamental, second, and third harmonics of magnetic induction $B$ versus $k=c / a$ for different intrinsic nonlinear characteristic $\xi H_{\mathrm{ac}}{ }^{2}$ according to eq 29.

between ferrogels (magnetism) and electrorheological fluids (electrics), we believe that the observation is qualitatively in agreement with our numerical results in Figures 1-6. In the figures, the intrinsic nonlinear characteristic $\xi H_{\mathrm{ac}}{ }^{2}$ is determined by the external magnetic field $H_{\mathrm{ac}}$ as well as the nonlinear coefficient $\xi$. Thus, an increasing nonlinear characteristic $\xi H_{\mathrm{ac}}{ }^{2}$ can arise from increasing $H_{\mathrm{ac}}$ and/or increasing $\xi$.

To simplify, our results present the fact that harmonics are closely related to elements such as the intrinsic nonlinear characteristics of particles, the volume fraction of nonlinear component, and the geometrical shape of particles. Therefore, it becomes possible to detect the volume fraction and shape of particles in ferrogels by measuring such ac responses.
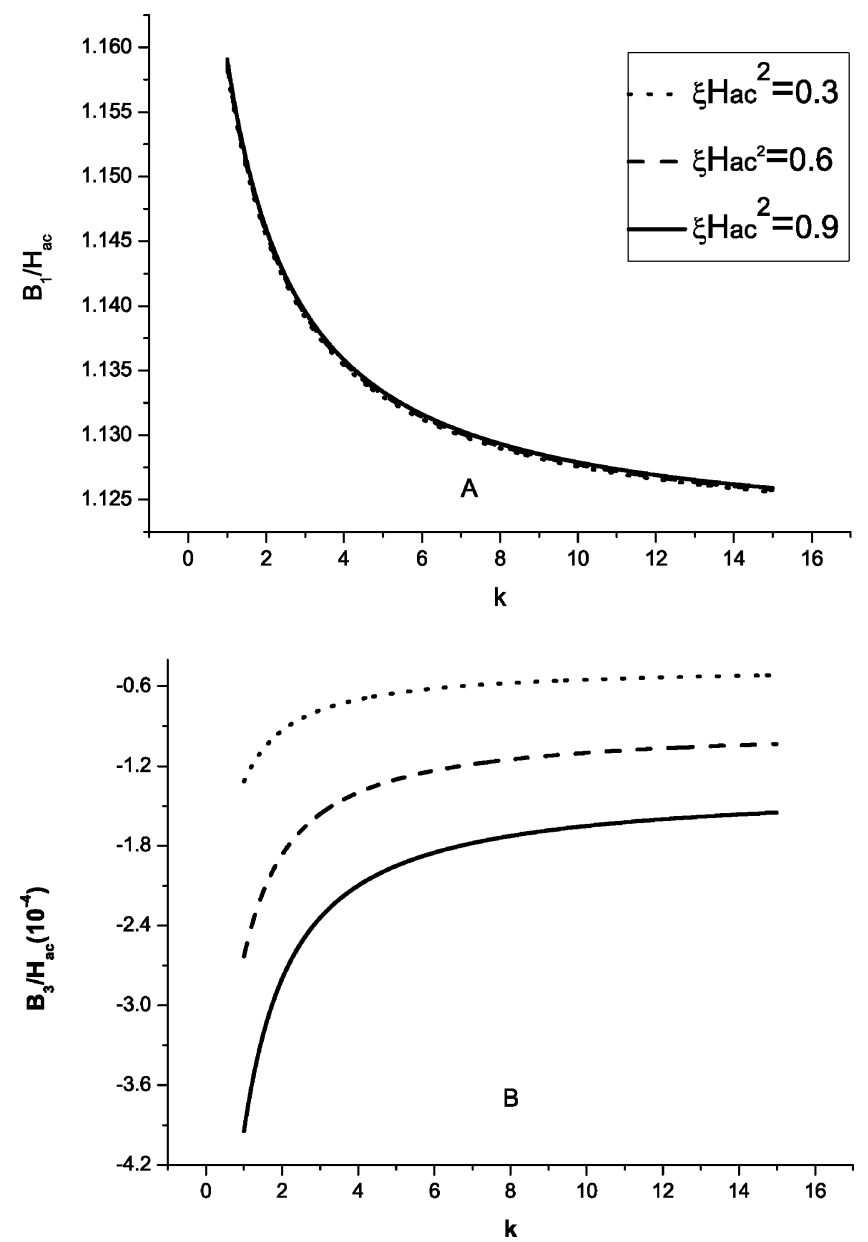

Figure 5. Cases of oblate spheroidal particles: ac field. Fundamental and third harmonics of magnetic induction $B$ versus $k=c / a$ for different intrinsic nonlinear characteristic $\xi H_{\mathrm{ac}}{ }^{2}$ according to eq 27.

\section{Discussion and Conclusion}

We have performed a perturbation approach to investigate the nonlinear ac responses (harmonics) of ferrogels, in an attempt to discuss the effects of the volume fraction and shape of particles in ferrogels. Throughout the paper (Figures 1-6), we have numerically calculated the harmonics up to the third order only. In fact, if higher harmonics such as the fifth order are calculated, similar results should be obtained as well. However, we should mention that the fifth harmonics are often of several orders of magnitude smaller than the third. Thus, the calculation of the third (or second) harmonics is more attractive than that of the fifth.

As a matter of fact, eq 2 contains only the third-order nonlinearity. In real situations under a stronger field, higherorder nonlinearities are possible, e.g.

$$
\mathbf{B}=\mu \mathbf{H}+\xi|H|^{2} \mathbf{H}+\eta|H|^{4} \mathbf{H}+\gamma|H|^{6} \mathbf{H}+\cdots
$$

where $\eta$ (or $\gamma$ ) is the fifth-order (or seventh-order) nonlinear coefficient. There must be $\gamma|H|^{6} \ll \eta|H|^{4} \ll \xi|H|^{2}$. In other words, the $\xi|H|^{2}$ (third-order nonlinearity) considered in this work represents the lowest-order nonlinearity which, however, has the strongest strength among the different nonlinearities. This is the reason we have studied the nonlinearity up to the third order only (eq 2). Here, we should further remark that higher harmonics can arise from different origins. Let us take fifth harmonics as an example. They can be induced to appear by the third-order nonlinearity. In other words, lower-order 

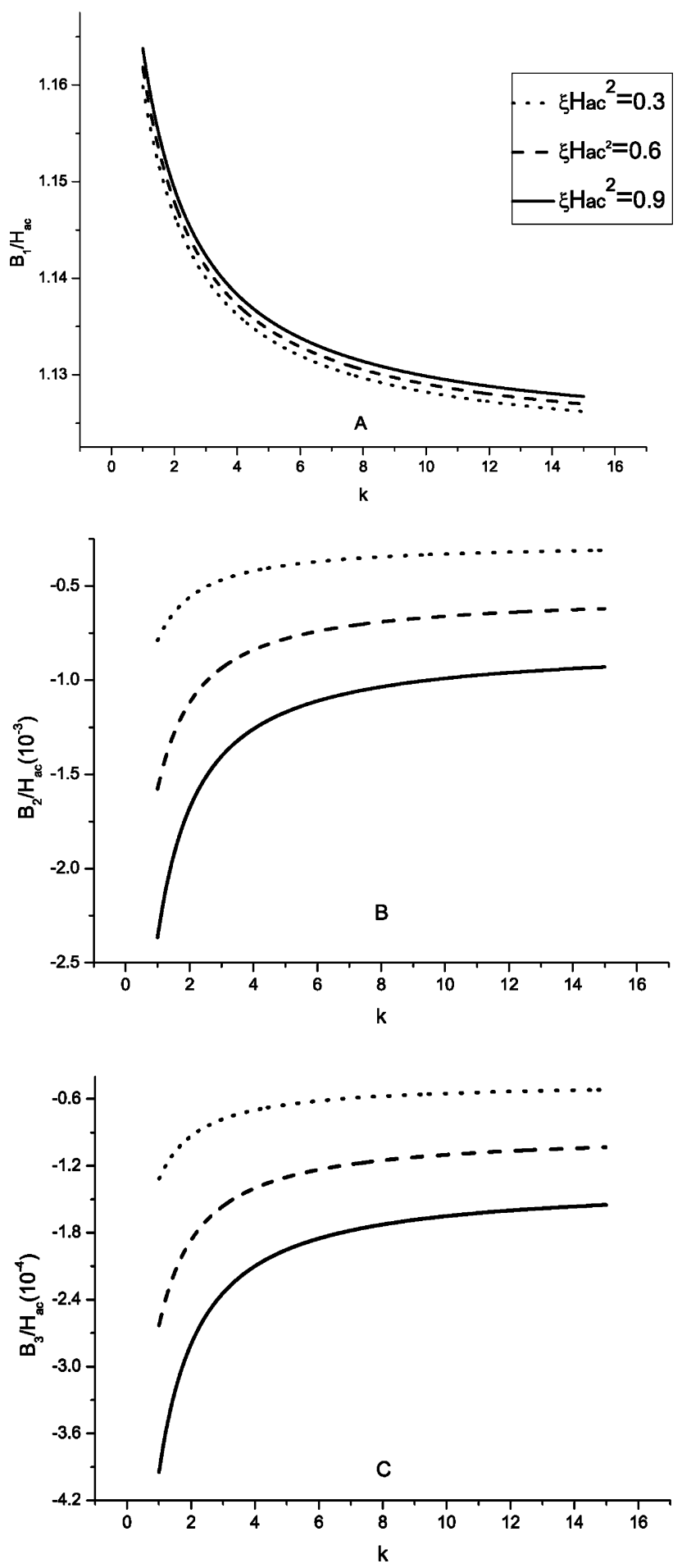

Figure 6. Cases of oblate spheroidal particles: ac and dc fields. Fundamental, second, and third harmonics of magnetic induction $B$ versus $k=c / a$ for different intrinsic nonlinear characteristic $\xi H_{\mathrm{ac}}{ }^{2}$ according to eq 29.

nonlinearity can induce higher-order nonlinear responses, as shown in the work (eqs 19, 20, 33, and 34). On the other hand, fifth harmonics can naturally be induced by the fifth-order nonlinearity $\eta|H|^{4}$ in eq 36 .

It is known that the volume fraction and the shape of fillers can be evaluated from magnetic measurements, e.g., saturation magnetization or initial magnetic susceptibility on a magnetization curve. In this work, we present an alternative method to do so by measuring nonlinear ac responses that arise from components with nonlinear characteristics that can exist in ferrogels. The nonlinearity under our consideration is weak, which is common in real situations under high fields. In case of strong nonlinearity, the perturbation approach is no longer valid. For this, a self-consistent method should be adopted instead. ${ }^{23,25}$

Since the magnetic susceptibility of water is $-9.0 \times 10^{-6}$, the relative magnetic permeability of water could be $1+(-9.0$ $\times 10^{-6}$ ) $\approx 1$. Even for the matrixes (namely, polymers and large amount of water), its magnetic susceptibility is only at most $10^{-5}$. In this case, the relative magnetic permeability could still be $1+\left(10^{-5}\right) \approx 1$. Thus, we took $\mu_{2}=1$ (magnetic permeability of the matrixes), which is both reasonable and practical. Regarding the suspended monodomain ferromagnetic nanoparticle, we took $\mu_{1}=6$. One invented a method to obtain cobalt (ferromagnetic component) sputter target with a magnetic permeability of less than about 9.28 Therefore, it is also reasonable to use $\mu_{1}=6$. On the other hand, even though one would take a $\mu_{1}$ that is much larger than $\mu_{2}$, the qualitative result we have achieved should be expected to remain unchanged, while quantitative results would be changed naturally.

To sum up, by using a perturbation approach, we have investigated nonlinear ac responses of ferrogels, under an ac magnetic field either coupled with a dc magnetic field or not. It is shown that it is possible to detect the volume fraction and shape of particles in ferrogels by measuring such ac responses.

Acknowledgment. We acknowledge the financial support by the Shanghai Education Committee and the Shanghai Education Development Foundation ("Shu Guang" project) under Grant No. KBH1512203, and by the Scientific Research Foundation for the Returned Overseas Chinese Scholars, State Education Ministry, China. This work was also supported by Jiangsu Key Laboratory of Thin Films, Suzhou University, China. We thank Professor K.W. Yu for his fruitful discussions.

\section{References and Notes}

(1) Brasi, L.; Zrinyi, M. ACH Models Chem. 1998, 135, 3.

(2) Zrínyi, M.; Horkay, F. J. Intell. Mater. Syst. Struct. 1993, 4, 190.

(3) Babincová, M.; Leszczynska, D.; Sourivong, P.; Čičmanec, P.; Babinec, P. J. Magn. Magn. Mater. 2001, 225, 109.

(4) Uritani M.; Hamada, A. Biochem. Educ. 1999, 27, 169. 40 .

(5) Zrinyi, M.; Brasi, L.; Büki, A. Europhys. Conf. Abstr. 1995, 19 G,

(6) Zrinyi, M.; Brasi, L.; Büki, A. J. Chem. Phys. 1996, 104, 21.

(7) Rosenweig, R. E. Ferrohydrodynamics; Cambridge University Press: Cambridge, 1985.

(8) Odenhach, S. Magnetoviscous Effects in Ferrofluids; Springer: Berlin, 2002.

(9) Jarkova, E.; Pleiner, H.; Müller, H. W.; Brand, H. R. Phys. Rev. E 2003, 68, 041706 .

(10) Zrinyi, M.; Brasi, L.; Szabo, D. J. Chem. Phys. 1997, 106, 13.

(11) Huang, J. P. J. Phys. Chem. B 2004, 108, 13901.

(12) Levy, O.; Bergman, D. J.; Stroud, D. Phys. Rev. E 1995, 52, 3184.

(13) Hui P. M.; Stroud, D. J. Appl. Phys. 1997, 82, 4740.

(14) Hui, P. M.; Cheung, P. C.; Stroud, D. J. Appl. Phys. 1998, 84, 3451 .

(15) Gu, G. Q.; Hui, P. M.; Yu, K. W. Physica B 2000, 279, 62.

(16) Wei, E. B.; Song, J. B.; Gu, G. Q. J. Appl. Phys. 2004, 95, 1377.

(17) Gu G. Q.; Yu, K. W. Phys. Rev. B, 1992, 46, 4502.

(18) Huang J. P.; Yu, K. W. J. Chem. Phys. 2004, 121, 15

(19) Stroud D.; Hui, P. M. Phys. Rev. B 1988, 37, 8719.

(20) Böttcher, C. J. F. Theory of Electric Polarization; 2nd ed.; Elsevier: Amsterdam, 1993; Vol. 1.

(21) Landau L. D.; Lifshitz, E. M.; Pitaevskii, L. P. Electrodynamics of Continuous Medium, 2nd ed.; Pergamon Press: New York, 1984; Chapter 2.

(22) Stoner, E. C. Philos. Mag. 1945, 36, 803.

(23) Huang, J. P.; Wan, J. T. K.; Lo, C. K.; Yu, K. W. Phys. Rev. E 2001, 64, 061505(R).

(24) Huang, J. P. Phys. Rev. E 2004, 70, 041403.

(25) Wan, J. T. K.; Gu, G. Q.; Yu, K. W. Phys. Rev. E 2001, 63, 052501.

(26) Bergman, D. J. Phys. Rep. 1978, 43, 377.

(27) Klingenberg D. J. MRS Bull. 1998, 23, 30.

(28) U.S. Patent 6,176,944, January 23, 2001. 